\title{
Nanoscale Cathodoluminescence of an InGaN Single Quantum Well Intersected by Individual Dislocations.
}

Gordon Schmidt, Peter Veit, Sebastian Metzner, Christoph Berger, Frank Bertram, Armin Dadgar, André Strittmatter, and Jürgen Christen.

Institute of Experimental Physics, Otto-von-Guericke-University Magdeburg, Germany.

Heteroepitaxially grown III-nitride quantum well layers contain a relatively high number of extended defects, like threading dislocations (TD), compared to III-arsenide ones. Generally, the non-radiative recombination at TDs is expected to reduce the efficiency of light emitting devices. Nevertheless, highly efficient light emitting devices based on InGaN/GaN quantum wells have been realized and commercialized since its first demonstration by S. Nakamura and co-workers. In contrast to the remarkable technical breakthroughs the physics of the active medium - the InGaN quantum wells remains not fully understood and is still debated.

Using cathodoluminescence spectroscopy directly performed in a scanning transmission electron microscope (STEM-CL), we analyze the optical properties of an InGaN single quantum well (SQW) with nanometer scale resolution close to individual threading dislocations. Embedded into GaN barriers the InGaN SQW was grown with an ammonia/group-III precursor (V/III-) ratio of 3000 at a temperature of $815^{\circ} \mathrm{C}$ by metal-organic vapor phase epitaxy on a GaN/sapphire template. To avoid the interdiffusion of $\mathrm{In}$ and $\mathrm{Ga}$ atoms in the InGaN/GaN heterostructure, the barrier material was grown at relatively low temperature of $935^{\circ} \mathrm{C}$.

Cross-sectional STEM images prove the comparably high structural quality of the GaN/sapphire template and the InGaN SQW in general. Originating from the buffer/sapphire interface vertically running TDs show up in the STEM images and intersect with the InGaN SQW. In dislocation free domains, the SQW layer with a thickness of $1.4 \pm 0.1 \mathrm{~nm}$ exhibits a sharp interface to the bottom GaN barrier while the upper interface has a diffuse contrast. The panchromatic CL with the highest intensity at $15 \mathrm{~K}$ is emitted by the SQW. The spatially averaged spectrum exhibits emission contributions from the SQW at $395 \mathrm{~nm}$ wavelength with distinct longitudinal optical phonon replica and near band edge emission from the $\mathrm{GaN}$ barriers at $357 \mathrm{~nm}$ wavelength.

With the high spatial resolution of our STEM-CL setup we are able to investigate the intersection of single dislocations with the InGaN SQW and its vicinity. Most strikingly, the SQW emission is blueshifted by up to $\Delta \mathrm{E}=70 \mathrm{meV}$ in the vicinity to $\mathrm{TDs}$ with concurrently reduced intensity. The detailed analysis of the evolution of the excitonic SQW luminescence across a single TD reveals an asymmetric emission profile. Directly exciting the SQW on one side of the TD, the SQW CL starts to shift continuously blue more than $200 \mathrm{~nm}$ apart from the SQW/TD intersection and reaches a minimum peak wavelength of $385 \mathrm{~nm}$ close to the intersection point. Tracking the SQW emission further across the TD, the peak wavelength continuously shifts to longer wavelengths up to $395 \mathrm{~nm}$ wavelength. The nature of the blue shift will be discussed in terms of local variations of strain, composition and thickness introduced by TDs. 

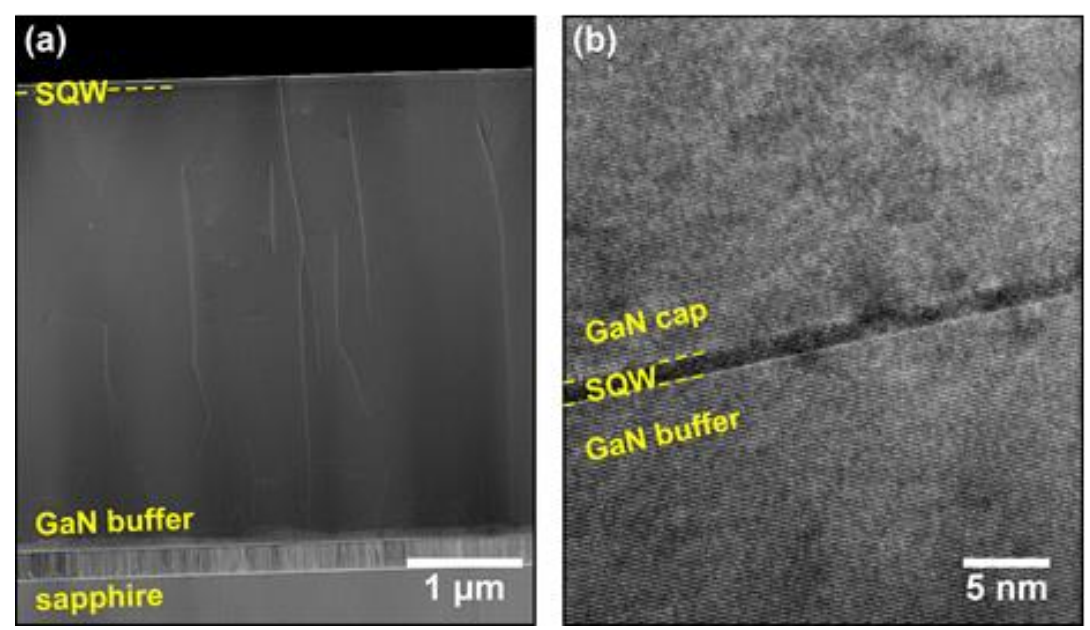

Figure 1. (a) HAADF image in overview of the sample, and (b) HRTEM micrograph of the $\mathrm{GaN} / \mathrm{InGaN} / \mathrm{GaN}$ quantum well heterostructure.



Figure 2. Cross-sectional STEM-CL mapping of the InGaN SQW with a single intersecting TD $(\mathrm{T}=16 \mathrm{~K}$ ): (a) HAADF image of the SQW region and the TD (marked by arrow), (b) SQW CL peak wavelength image, monochromatic CL intensity images from (c) high energy SQW emission and (d) SQW main emission, (e, f) local spectra; (TD marked as dash-dot-line and vacuum/sample interface marked as dashed line in $(b-d)$. 